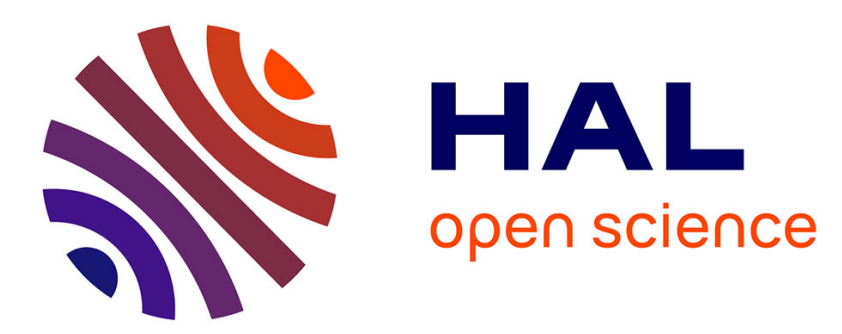

\title{
Critical exponents of two-dimensional Potts and bond percolation models
}

\author{
H Blote, M Nightingale, Bernard Derrida
}

\section{To cite this version:}

H Blote, M Nightingale, Bernard Derrida. Critical exponents of two-dimensional Potts and bond percolation models. Journal of Physics A: Mathematical and General, 1981, 14 (2), pp.L45-L49. 10.1088/0305-4470/14/2/005 . hal-03285937

\section{HAL Id: hal-03285937 \\ https://hal.science/hal-03285937}

Submitted on 19 Jul 2021

HAL is a multi-disciplinary open access archive for the deposit and dissemination of scientific research documents, whether they are published or not. The documents may come from teaching and research institutions in France or abroad, or from public or private research centers.
L'archive ouverte pluridisciplinaire HAL, est destinée au dépôt et à la diffusion de documents scientifiques de niveau recherche, publiés ou non, émanant des établissements d'enseignement et de recherche français ou étrangers, des laboratoires publics ou privés. 


\title{
LETTER TO THE EDITOR
}

\section{Critical exponents of two-dimensional Potts and bond percolation models}

\author{
H W J Blöte†, M P Nightingale $\dagger$ and B Derrida \\ † Laboratorium voor Technische Natuurkunde, Technische Hogeschool Delft, Delft, The \\ Netherlands \\ ¥ Service de Physique Théorique, CEN Saclay, Gif-sur-Yvette, France
}

Received 27 October 1980

\begin{abstract}
Critical exponents of the two-dimensional, $q$-state Potts model are calculated by means of finite size scaling and transfer matrix techniques for continuous $q$. Results for the temperature exponent agree accurately with the conjecture of den Nijs. The magnetic exponent is found to behave in accordance with the conjecture of Nienhuis et al.
\end{abstract}

It has been shown (Nightingale 1977, 1979, Sneddon 1978, Nightingale and Blöte 1980 , Roomany et al 1980 , and references therein) that finite size scaling in conjunction with the transfer matrix technique for the calculation of properties of finite systems provides a powerful method to calculate critical exponents. A particular implementation of this idea was applied to the $q$-state Potts model on a quadratic lattice. In order to obtain the free energy of a $n \times \infty$ Potts model, the $q^{n} \times q^{n}$ transfer matrix was straightforwardly constructed for $q=2,3,4$ and 5 , after which its largest eigenvalue was calculated. The specific heat and susceptibility were evaluated by means of numerical differentiation. A comparison with known scaling relations for finite systems produced estimates of the desired critical point exponents. The results (Nightingale and Blöte 1980) were in good agreement with rigorous and conjectured results for $q=2$ and 3 respectively. It was demonstrated that the exponents could be accurately determined from data on small systems with linear sizes up to about ten. However, this procedure does not work for the percolation (Wu 1978) problem $(q \rightarrow 1)$, while large $q$ also cannot be handled. In the present formulation $q$ enters as a continuously variable parameter only.

Consider a simple quadratic lattice consisting of $n$ horizontal rows and $m$ vertical columns; $n$, the width of the system, will be treated as fixed. At the lattice sites one has Potts variables $\sigma_{i}=1, \ldots, q$, with a nearest-neighbour coupling $K$. In zero field the partition function reads

$$
Z_{m}=\sum_{\sigma_{1}} \ldots \sum_{\boldsymbol{\sigma}_{m}} \exp \left(K \sum_{(i, j)} \delta_{\sigma_{i} \sigma_{j}}\right)
$$

where $\boldsymbol{\sigma}_{j}=\left(\sigma_{1 j}, \ldots, \sigma_{n j}\right)$ runs through all states of column $j$, while $(i, j)$ runs through all nearest-neighbours pairs. The boundary conditions in the vertical directions are arbitrary; horizontally we assume free boundaries. The partition function (1) may be 
written (Kasteleyn and Fortuin 1969, Baxter 1973) as a Whitney (1932) polynomial

$$
Z_{m}=q^{n m} \sum_{G \in \mathscr{L}_{m}}\left(\frac{u}{q}\right)^{b(G)} q^{l(G)}
$$

where $u \equiv \exp (K)-1$, and the sum is over all graphs $G$ on the lattice $\mathscr{L}_{m} ; b(G)$ and $l(G)$ respectively are the number of bonds and independent loops in $G$. Expression (2) is the partition function of a system of interacting bond variables with Hamiltonian

$$
\mathscr{H}^{\prime}(G)=n m \ln (q)+b(G) \ln (u / q)+l(G) \ln (q) .
$$

As it stands, this Hamiltonian is not a sum of column-column contributions. Therefore, the transfer matrix in its usual form cannot be applied. However, the desired additivity property can be obtained as follows. Consider a graph $F$ on the lattice $\mathscr{L}_{m+1}$ which is the original lattice extended by one column. The graphs $F$ can be split into two parts $F=G \cup g$, where $G$ is $F$ restricted to $\mathscr{L}_{m}$ and $g$ is that part of $F$ containing bonds between the columns $m$ and $m+1$ and bonds within column $m+1$. Graph $G$ on $\mathscr{L}_{m}$ defines a state of connectivity $\alpha$ of the sites on column $m$. Specifically, we define $\alpha=\left(i_{1}, i_{2}, \ldots, i_{n}\right)$, where the $i_{k}$ are integers such that: (i) $i_{j}=i_{k}$ if and only if there is a path in $G$ connecting the sites $j$ and $k$ of column $m$; (ii) $i_{1}=1$, and either $i_{j+1}=\max \left(i_{1}, \ldots, i_{j}\right)+1$ or $i_{j+1}=i_{k}$ for some $k \leqslant j$. Clearly, if $i_{j}=i_{l}$ and $i_{k}=i_{m}$ with $j<k<l<m$, then also $i_{l}=i_{k}$; the integers in the sequence $\alpha$ are referred to as being well nested. With the help of the splitting $F=G \cup g$, one has

$$
\left.\begin{array}{l}
\alpha(F) \text { depends on } \alpha(G) \text { and } g \text { only } \\
\mathscr{H}^{\prime}(F)=\mathscr{H}^{\prime}(G)+\Delta \mathscr{H}^{\prime}(\alpha(G), g)
\end{array}\right\}
$$

since $\alpha(G)$ and $g$ together determine the increase in the number of bonds and loops due to adding column $m+1$, as well as the state of connectivity of this column. To define the transfer matrix, one introduces the constrained partition function

$$
Z_{m}(\alpha)=\sum_{G \mid \alpha} \exp (\mathscr{H})
$$

where the summation is over all graphs $G$ compatible with a state of connectivity $\alpha$ of column $m$. One obtains, using property (4),

$$
Z_{m+1}(\beta)=\sum_{F \mid \beta} \exp \left(\mathscr{H}^{\prime}\right)=\sum_{\alpha}\left\{\sum_{g \mid \beta \alpha} \exp \left[\Delta \mathscr{H}^{\prime}(\alpha, g)\right]\right\} Z_{m}(\alpha)
$$

where the second summation is over all partial graphs $g$ compatible with columns $m$ and $m+1$ being in states $\alpha$ and $\beta$ respectively. The factor in braces depends on $\alpha$ and $\beta$ only, and defines the transfer matrix T. As usual, its largest eigenvalue $\lambda_{0}$ is related to the free energy per site $f=-(k T / n) \ln \lambda_{0}$ in the limit $m \rightarrow \infty$. Since $q$ enters as a parameter only, the present calculations are no longer restricted to integral $q$.

Temperley and Lieb (1971) have shown how the transfer matrix of a Whitney polynomial can be expressed in spin operators. We have not used this formulation. Instead, we constructed a fast algorithm for the transfer matrix multiplication, directly using connectivities, and writing $\mathbf{T}=\mathbf{V}^{n} \cdot \mathbf{H}^{n}$ where $\mathbf{V}$ (or $\mathbf{H}$ ) is a matrix which corresponds to adding one vertical (horizontal) bond variable at a time. Our approach is close in spirit to the one used by Derrida and Vannimenus (1980) for the percolation problem. Technical details concerning the calculation of $\lambda_{0}$ will be published elsewhere. 
For the calculation of the magnetic exponent we define

$$
\mathscr{H}=K \sum_{(i, j)} \delta_{\sigma_{i} \sigma_{j}}+h \sum_{(i, 0)} \delta_{\sigma_{i} \sigma_{0}}
$$

where we have introduced a ghost site labelled 0 , with Potts variable $\sigma_{0}=1$, and interacting with all other spins via the bonds $(i, 0)$. In order to write the partition function of (7) as a sum over graphs, we introduce the following notation. By $\mathscr{L}_{m}^{\prime}=\mathscr{L}_{m} \cup \mathscr{L}_{m}^{0}$ we denote the $m$-column lattice $\mathscr{L}_{m}$ extended by $\mathscr{L}_{m}^{0}$, which stands for the ghost site and its bonds. Accordingly, each graph $G^{\prime}$ on the lattice $\mathscr{L}_{m}^{\prime}$ can be split into two parts, $G^{\prime}=G \cup G^{0}$, where $G$ and $G^{0}$ are subgraphs of $\mathscr{L}_{m}$ and $\mathscr{L}_{m}^{0}$ respectively. The partition function of $(7)$ can now be written as

$$
Z_{m}=q^{n m} \sum_{G^{\prime}}\left(\frac{u}{q}\right)^{b(G)}\left(\frac{v}{q}\right)^{b\left(G^{0}\right)} q^{l\left(G^{\prime}\right)}
$$

where $v \equiv \exp (h)-1$. A transfer matrix for expression (8) can be defined as before, provided that we modify the definition of the connectivities $\alpha^{\prime}=\left(i_{1}, \ldots, i_{n}\right)$ such that the $i_{k}$ are now also allowed to assume the value $0 ; i_{k}=0$ whenever site $k$ is connected to the ghost site by some path. The well-nestedness property now holds for integers $i_{k}>0$ only. Since the number of connectivities $\alpha^{\prime}$ is considerably larger than the number of connectivities $\alpha$ for given $n$, calculations are restricted to smaller $n$ when a field is included. For $h<0$ expression (8) contains negative terms. Thus for general $q$ it can no longer be directly interpreted as a partition function, and (8) may become unphysical. We have observed that for $q<1$ the largest eigenvalue of $\mathbf{T}$ may become complex for negative $h$. However, for sufficiently small $|h|$ the eigenvalue is real and behaves smoothly as a function of $h$.

Temperature exponents were obtained from heat capacity data on infinitely long strips with widths up to $n=10$ and periodical boundaries in the finite direction. Extrapolation to $n=\infty$ was performed in two different ways: (i) Padé approximants in $1 / n$ to estimates $y_{n}$ of the exponent $y_{\infty}$ (Nightingale and Blöte 1980); (ii) extrapolations based on the assumption that $y_{\infty}-y_{n}=a(q) b(n)$ and exact results for $y_{\infty}$ at $q=2$. May it suffice to note that these methods display a high degree of internal consistency and yield an estimate of the accuracy. The value of $y_{\mathrm{T}}$ at $q=1$ was found by interpolation between values obtained close to $q=1$. Results are shown in figure 1 together with the conjectured exact formula of den Nijs (1979): $y_{T}=3-3(2-2 p)$ with $p=$ $\cos ^{-1}(\sqrt{ } q / 2) / \pi$. Differences between our data points and this expression are about $10^{-3}$ or smaller for $q<3$, not visible on the scale of figure 1 . These small differences are of the same order as the estimated accuracy of our method. For $q \approx 4$ slow convergence may be attributed to the presence of a marginal eigenvalue as argued previously (Nightingale and Blöte 1980). The excellent agreement suggests that the conjecture of den Nijs is correct (cf Burkhardt 1980). It is also noteworthy that our result for $q=1$, i.e. $y_{\mathrm{T}}=0.750 \pm 0.001$, excludes a conjectured exact value given by Klein et al (1978).

The magnetic exponent $y_{h}$ was similarly obtained from susceptibility data on strips with widths up to 8 . Results are plotted in figure 2 , together with the conjectured exact formula of Nienhuis et al (1980): $y_{h}=\frac{3}{2}+3 /(8-8 p)-p / 2$ with $p$ given above. The data points agree with this conjecture within the estimated accuracy of our method $(\sim 0.001$ on the $y_{h}$ scale for $q<3$ ).

Calculation of the energy for $q \gg 4$ (up to 64) gave results rapidly approaching a step function with increasing $n$, in accordance with a first-order transition (Baxter 1973). Fitted exponents were found to increase approximately linearly with system size, 


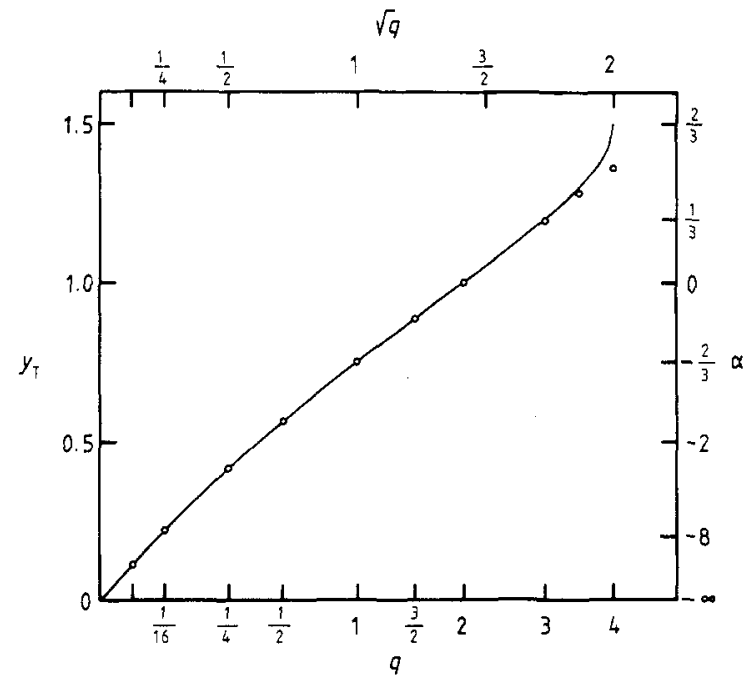

Figure 1. Temperature exponent $y_{\mathrm{T}}$ of the Potts model according to the conjecture (full curve) of den Nijs (1979) and the present results (data points). Agreement is very good except near the point $q=4$ (cf Nightingale and Blöte 1980).

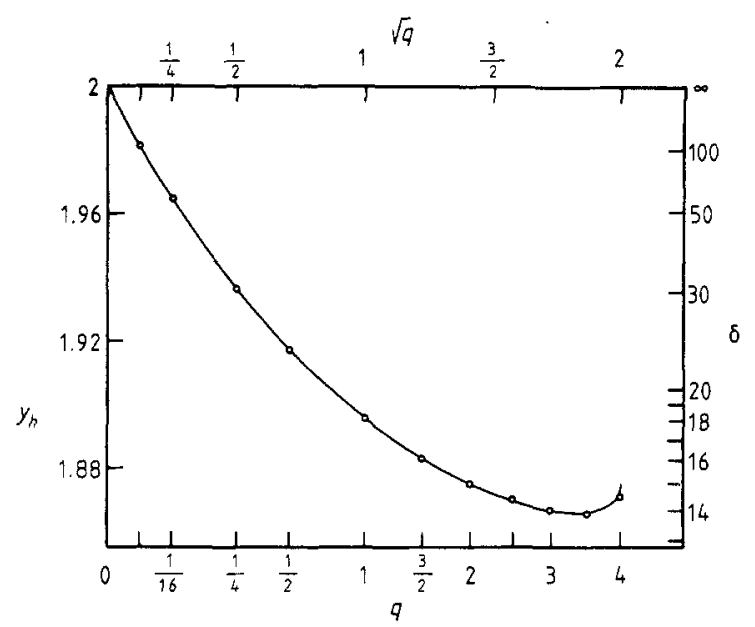

Figure 2. Magnetic exponent $y_{h}$ of the Potts model according to the conjecture (full curve) of Nienhuis et al (1980) and the present results (data points). Agreement is very good except near the point $q=4$ (cf Nightingale and Blöte 1980).

corresponding to exponential divergence of specific heat with $n$ and exponential convergence of the energy to a step function. Similar divergent behaviour for the susceptibility was observed.

We are indebted to Professor J M J van Leeuwen, Dr V J Emery and Dr H J Hilhorst for valuable discussions. These investigations form part of the research programme of the 'Stichting voor Fundamenteel Onderzoek der Materie (FOM)' which is financially supported by the 'Nederlandse Organisatie voor Zuiver-Wetenschappelijk Onderzoek (ZWO)'. 


\section{References}

Baxter R J 1973 J. Phys. C: Solid State Phys. 6 L455-8

Burkhardt Th W 1980 Z. Phys. B 39 159-62

Derrida B and Vannimenus J $1980 \mathrm{~J}$. Physique Lett. 41 473-6

Kasteleyn P W and Fortuin C M 1969 J. Phys. Soc. Japan 26 (suppl.) 11-4

Klein W, Stanley H E, Reynolds P J and Coniglio A 1978 Phys. Rev. Lett. 41 1145-8

Nienhuis B, Riedel E K and Schick M 1980 J. Phys. A: Math. Gen. 13 L189-92

Nightingale M P 1977 Phys. Lett. 59A 486-8

1979 Proc. Kon. Ned. Ak. Wet. B 82 235-91

Nightingale M P and-Blöte H W J 1980 Physica A 104 352-7

den Nijs M P M 1979 J. Phys. A: Math. Gen. 12 1857-68

Roomany H H, Wyld H W and Holloway L E 1980 Phys. Rev. D21 1557-63

Sneddon L 1978 J. Phys. C: Solid State Phys. 11 2823-8

Temperley H N V and Lieb E H 1971 Proc. R. Soc. Lond. A322 251-80

Whitney H 1932 Ann. Math., NY 33 688-718

Wu F Y 1978 J. Statist. Phys. 18 115-23 